\title{
DESEMPENHO DO CAMARÃO Macrobrachium amazonicum (HELLER, 1862) (CRUSTACEA: DECAPODA: PALAEMONIDAE), EM DIFERENTES DENSIDADES
}

\author{
Igor da Silva MACHADO ${ }^{1}$; Cesar Antunes Rocha NUNES ${ }^{1}$; Helder Batista Lopes dos SANTOS ${ }^{1}$; \\ Jefferson Alves LIMA ${ }^{1}$; Tarcisio Martins MEIRA ${ }^{1}$; Edimilson da Silva SENA ${ }^{1}$ \& Wennie Nunes \\ da SILVA ${ }^{1}$ \\ ${ }^{1}$ Departamento de Ciências Humanas e Tecnologias, Universidade do Estado da Bahia - UNEB \\ *e-mail: igor.s.machado@ hotmail.com \\ Recebido em: 19/12/2017
}

Resumo - O cultivo de camarão de água doce tornou-se um dos principais setores do agronegócio, contribuindo para o crescimento da aquicultura mundial. $\mathrm{O}$ presente trabalho teve como objetivo avaliar o desempenho do camarão Macrobrachium amazonicum em diferentes densidades de estocagem. O experimento foi realizado na Universidade do Estado da Bahia (UNEB), no Departamento de Ciências Humanas e Tecnologias (DCHT XXIV), Campus Xique-Xique, BA. Os camarões foram aclimatados em tanques tipo caixa d'água de polietileno de 5001 durante sete dias. Posteriormente foram selecionados e transferidos para 20 tanques experimentais de $20 \mathrm{l}$, com sistema de recirculação de água, filtros biológico e físico, e aeração constante. Utilizou-se um delineamento experimental inteiramente casualizado, com cinco tratamentos e quatro repetições, totalizando 20 parcelas experimentais. No início do estudo, os camarões foram selecionados, com peso médio de $0,54 \pm 0,26 \mathrm{~g}$, submetidos a tratamentos com densidades de 20, 40, 60, 80 e 100 camarões $/ \mathrm{m}^{2}$. A alimentação foi feita diariamente com ração comercial peletizada com conteúdo proteico de $35 \%$ a $4 \%$ da biomassa. Os resultados foram submetidos à utilização do programa estatístico SAS versão 9.1. Os valores médios das variáveis físico-químicas de qualidade da água não tiveram diferença significativa entre os cinco tratamentos. Ao avaliar o efeito da densidade sobre sobrevivência $(100,00 ; 75,00 ; 50,00 ; 58,33 ; 40,00 \%)$ e peso médio $(0,63 ; 1,08 ; 1,63 ; 2,28$; $2,52 \mathrm{~g}$ ), foi observada diferença significativa entre os tratamentos. Já o ganho de peso, biomassa final e produtividade não apresentaram diferença significativa entre as densidades estudadas. Foi observada uma relação positiva com crescimento linear entre o peso médio do cultivo e a densidade. O cultivo de $M$. amazonicum é viável utilizando densidade entre 80 e 100 camarões $/ \mathrm{m}^{2}$ sem prejuízo do desempenho zootécnico, favorecendo a produção da espécie em cultivos com altas densidades.

Palavras-chave: carcinicultura, camarão de água doce, desempenho, estocagem.

\section{PERFORMANCE OF SHRIMP MACROBRACHIUM AMAZONICUM (HELLER, 1862) (CRUSTACEA: DECAPODA: PALAEMONIDAE), IN DIFFERENT DENSITIES}

Abstract - The cultivation of freshwater shrimp has become one of the main sectors of agribusiness, contributing to the growth of world aquaculture. The objective of this work was to evaluate the performance of Macrobrachium amazonicum shrimp at different storage densities. The experiment was carried out at the State University of Bahia (UNEB), at the Department of Human Sciences and Technologies (DCHT XXIV), in Xique-Xique Campus, Bahia. The shrimps were acclimatized in 500-liter polyethylene water tanks for seven days. Subsequently they were selected and transferred to 20 experimental tanks of $20 \mathrm{~L}$, with water recirculation system, biological and physical filters, and constant aeration. A completely randomized experimental design was used, with five treatments and four replications, totalizing 20 experimental plots. At the beginning of the study, the shrimp were selected with an average weight of $0.54 \pm 0.26 \mathrm{~g}$, and submitted to treatments with densities of $20,40,60,80$ and $100 \mathrm{shrimp} / \mathrm{m}^{2}$. There was daily feeding with commercial pelleted feed with a protein content of 35 at $4 \%$ of the biomass. The results were submitted to the statistical program SAS version 9.1. The mean values of the physicochemical variables of water quality did not have significant differences between the five 
treatments. A significant difference between treatments was observed when evaluating the effect of density on survival $(100.00,75.00,50.00,58.33,40.00 \%)$ and mean weight $(0.63,1.08,1.63,2.28$, $2.52 \mathrm{~g})$. However, the weight gain, final biomass and productivity did not present a significant difference between the studied densities. A positive relation with linear growth was observed, between the average weight of the crop and their density. The cultivation of M. amazonicum is feasible using density between 80 and 100 shrimp $/ \mathrm{m}^{2}$ without prejudice to the zootechnical performance, favoring the production of the species in high density cultures.

Keywords: carciniculture, freshwater shrimp, performance, storage. 


\section{INTRODUÇÃO}

A aquicultura mundial produziu mais de 73,8 milhões de toneladas em 2014, sendo 6,9 milhões de crustáceos. Esse número representa 9,3\% do total e gerou um lucro de 36,2 bilhões de dólares (FAO, 2016).

O cultivo de camarão de água doce tornou-se um dos principais fatores para o crescimento da aquicultura mundial. Entre as espécies mais cultivadas estão a Macrobrachium nipponense (De Haan, 1849), produzida principalmente na China, e a Macrobrachium rosenbergii (De Man, 1879), cultivada em vários países, principalmente os asiáticos (New, 2010).

Em 2015 a produção da aquicultura brasileira foi de 574,164 t, gerando capital de 4,39 bilhões de reais, sendo $69,9 \%$ representado pela piscicultura e $20,6 \%$ pela carcinicultura. A produção de camarão foi de 69.859 t, com um crescimento de 7,45\% em relação a 2014 (IBGE, 2015).

Nos últimos anos, a carcinicultura tem apresentado um grande crescimento, principalmente nos países emergentes, desempenhando um grande papel socioeconômico com geração de renda em esfera regional e nacional.

No mercado mundial da aquicultura, o camarão se destaca como um dos principais produtos pesqueiros, devido ao seu grande valor econômico, além de ter um elevado teor nutritivo e grande aceitação pelos consumidores (Moraes-Valenti \& Valenti, 2010).

Na América do Sul, a espécie nativa com maior potencial é a Macrobrachium amazonicum (Heller, 1862), que possui características fundamentais como rusticidade, resistência a doenças e alta taxa de sobrevivência, o que resulta em um bom desempenho zootécnico e grande aceitação comercial (Maciel \& Valenti, 2009). Trata-se da espécie ideal para cultivo, com um grande potencial para carcinicultura.

A contribuição de espécies nativas em empreendimentos aquícolas está de acordo com práticas de sustentabilidade e gestão ambiental, uma vez que amenizam os riscos gerados pelo escape acidental dos camarões no meio ambiente (Marques \& Moraes-Valenti, 2012). Assim, potencializa-se ainda mais o cultivo da espécie e coopera-se com a liberação para implementação de novos cultivos.

No Brasil, a carcinicultura de água doce utiliza, na maior parte dos empreendimentos, a espécie exótica Macrobrachium rosenbergii, que pode causar grandes impactos ambientais e riscos de surgimento de doenças, fazendo com que as espécies nativas tenham que competir por seu espaço (Araújo \& Valenti, 2005). Por esse motivo faz-se necessário o desenvolvimento de pacotes tecnológicos para espécies nativas, a fim de amenizar os impactos ambientais. 
A densidade de estocagem de camarões é um dos principais fatores que determinam o crescimento, podendo variar em função da competição por alimento e espaço. Altas densidades elevam o aumento de segregação, canibalismo, competição por alimento e espaço, e consequentemente de sobrevivência e da biomassa final. Porém, baixas densidades inviabilizam o empreendimento, podendo variar em função da espécie utilizada no cultivo e também das estratégias de manejo de cultivo ou de parâmetros ambientais (Krummenauer et al., 2006).

Diante da carência de estudos para o cultivo de $M$. amazonicum, o presente trabalho tem o objetivo de avaliar o desempenho do camarão $M$. amazonicum em diferentes densidades de estocagem.

\section{MATERIAis E Métodos}

O experimento foi realizado na Universidade do Estado da Bahia (UNEB), no Departamento de Ciências Humanas e Tecnologias (DCHT XXIV), Laboratório de Carcinicultura, Campus XiqueXique, município de Xique-Xique, Bahia. Foram utilizados juvenis de camarões da espécie $M$. amazonicum, capturados utilizando puçá, nos horários noturnos, das 18 às 20 horas, localizados no braço do Rio São Francisco (Ipueira), às margens da sede do município de Xique-Xique.

Os camarões foram aclimatados em tanques tipo caixa d'água de polietileno de 500 litros, durante sete dias. Logo após a aclimatação, foram selecionados e transferidos para os tanques experimentais, com sistema de recirculação de água fechado, composto por 20 garrafões de 20 litros confeccionados de policarbonato, material higiênico, atóxico e reciclável, com filtro biológico e físico, e aeração constante.

O delineamento experimental utilizado foi inteiramente casualizado, com cinco tratamentos e quatro repetições, totalizando 20 parcelas experimentais. No início do estudo, os camarões selecionados (80 juvenis), com peso médio de 0,54 $\pm 0,26 \mathrm{~g}$, foram submetidos a tratamentos com densidades de 20, 40, 60, 80 e 100 camarões $/ \mathrm{m}^{2}$, sendo alimentados durante sete dias antes do início da coleta de dados, para adaptação às rações e ao manejo experimental. $\mathrm{O}$ arraçoamento foi realizado duas vezes por dia, utilizando ração comercial peletizada com 35\% de proteína, nos horários de 8 e 17 horas, durante 30 dias.

Foram realizadas sifonagens duas vezes por dia, antes dos arraçoamentos, com retirada de cerca de $10 \%$ do volume do tanque e reposição com água proveniente da rede pública, reservada em um tanque de polietileno de $500 \mathrm{~L}$, permanecendo por $24 \mathrm{~h}$ com aeração constante para volatilização do cloro.

As biometrias foram feitas semanalmente, pesando $100 \%$ do lote (peso úmido) em balança eletrônica de precisão $(0,01 \mathrm{~g})$, para obter o peso médio das repetições e de sua biomassa. 
Inicialmente, as dietas foram fornecidas em uma taxa de $4 \%$ da biomassa dos camarões de cada repetição, fazendo ajustes de acordo com o consumo. Logo após a pesagem, os camarões eram devolvidos aos seus tanques de origem.

Os parâmetros físico-químicos como $\mathrm{pH}$, oxigênio dissolvido, temperatura, salinidade e condutividade elétrica foram aferidos duas vezes ao dia, às 8 e 17 horas, por meio da sonda multiparâmetros HANNA HI 9828.

No fim do período experimental, os camarões foram contados e pesados para análise dos parâmetros zootécnicos por meio das seguintes variáveis: peso médio ( $\mathrm{PM}=$ peso total dos camarões / número total de camarões); sobrevivência (Sob\% = número final de camarões $\times 100$ / número inicial de juvenis); ganho de peso ( $\mathrm{GP}=$ peso final - peso inicial); biomassa total $(\mathrm{BT}=$ peso médio $\times$ número de camarões) e produtividade (prod. = kg camarão/ha).

Para as variáveis físico-químicas de qualidade de água e de desempenho zootécnico, testou-se a atividade por meio do procedimento General Linear Models (GLM), utilizando-se a análise de covariância dos valores preditos ao quadrado. A normalidade foi testada pelo procedimento "univariate", por meio da estatística W (Shapiro-Wilk). A homogeneidade de variância foi avaliada pelo teste de Barlett e as diferenças foram detectadas pelo teste de Tukey a 5\% de probabilidade (p $\leq$ 0,05), com a utilização do programa estatístico SAS versão 9.1 (SAS, 1999).

\section{RESULTADOS}

Os valores médios das variáveis físico-químicas de qualidade da água (Tabela 1) não tiveram diferença significativa $(\mathrm{p}>0,05)$ entre os cinco tratamentos. Os parâmetros físico-químicos da água de cultivo variaram dentro dos níveis aceitáveis para a produção de camarões de água doce.

Tabela 1. Valores médios das variáveis físico-químicas de qualidade da água do cultivo de Macrobrachium amazonicum submetido a diferentes densidades de estocagem.

\begin{tabular}{ccccccc}
\hline V. Físico-químicas & $20 \mathrm{cam} / \mathrm{m}^{2}$ & $40 \mathrm{cam} / \mathrm{m}^{2}$ & $60 \mathrm{cam} / \mathrm{m}^{2}$ & $80 \mathrm{cam} / \mathrm{m}^{2}$ & $100 \mathrm{cam} / \mathrm{m}^{2}$ & $\mathrm{CV}(\%)$ \\
\hline Temperatura $\left({ }^{\circ} \mathrm{C}\right)$ & 29,46 & 29,42 & 29,45 & 29,46 & 29,52 & 0,23 \\
$\begin{array}{c}\text { Condutividade } \\
(\mu \mathrm{S} / \mathrm{cm})\end{array}$ & 184,94 & 185,22 & 184,99 & 184,78 & 185,02 & 0,20 \\
$\begin{array}{c}\text { Oxigênio dissolvido } \\
(\mathrm{mg} / \mathrm{L})\end{array}$ & 3,25 & 3,23 & 3,24 & 3,22 & 3,22 & 0,59 \\
$\mathrm{pH}$ & & & & & & \\
Salinidade (ppt) & 0,20 & 9,00 & 9,09 & 9,14 & 9,12 & 0,75 \\
\hline
\end{tabular}

Cam: camarão; CV: coeficiente de variação.

O ganho de peso, biomassa final e produtividade não apresentaram diferença significativa 
( $p>0,05)$ entre as densidades estudadas. Ao avaliar o efeito da densidade sobre sobrevivência e do peso médio, observou-se diferença significativa $(\mathrm{p}<0,05)$ entre os tratamentos (Tabela 2$)$.

Tabela 2. Valores médios de desempenho de juvenis de Macrobrachium amazonicum em cinco tratamentos de diferentes densidades de estocagem.

\begin{tabular}{ccccccc}
\hline Variáveis & $20 \mathrm{cam} / \mathrm{m}^{2}$ & $40 \mathrm{cam} / \mathrm{m}^{2}$ & $60 \mathrm{cam} / \mathrm{m}^{2}$ & $80 \mathrm{cam} / \mathrm{m}^{2}$ & $100 \mathrm{cam} / \mathrm{m}^{2}$ & $\mathrm{CV}(\%)$ \\
\hline Peso médio $(\mathrm{g})$ & $0,63 \mathrm{C}$ & $1,08 \mathrm{BC}$ & $1,63 \mathrm{ABC}$ & $2,28 \mathrm{AB}$ & $2,52 \mathrm{~A}$ & 23,87 \\
Sobrevivência (\%) & $100,00 \mathrm{~A}$ & $75,00 \mathrm{AB}$ & $50,00 \mathrm{AB}$ & $58,33 \mathrm{AB}$ & $40,00 \mathrm{~B}$ & 33,24 \\
Ganho de Peso (g) & 0,25 & 0,45 & 0,53 & 0,46 & 0,08 & 54,49 \\
Biomassa final (g) & 0,75 & 1,30 & 1,68 & 2,30 & 1,54 & 43,35 \\
Produtividade (g/m) & 13,10 & 15,29 & 17,86 & 18,36 & 13,50 & 19,35 \\
\hline
\end{tabular}

Médias seguidas pela mesma letra nas linhas não diferem pelo teste de Tukey ( $p>0,05$ ); cam: camarão; $\mathrm{CV}$ : coeficiente de variação.

Verificou-se que o aumento da densidade não influenciou significativamente o ganho de peso, produtividade e biomassa final em todos os tratamentos, sendo que a biomassa final obteve os melhores valores no tratamento de $80 \mathrm{cam} / \mathrm{m}^{2}$ (Tabela 2).

Apesar disso, o aumento da densidade não configurou redução do ganho de peso, com ganho positivo em relação ao aumento das densidades (Figura 1).

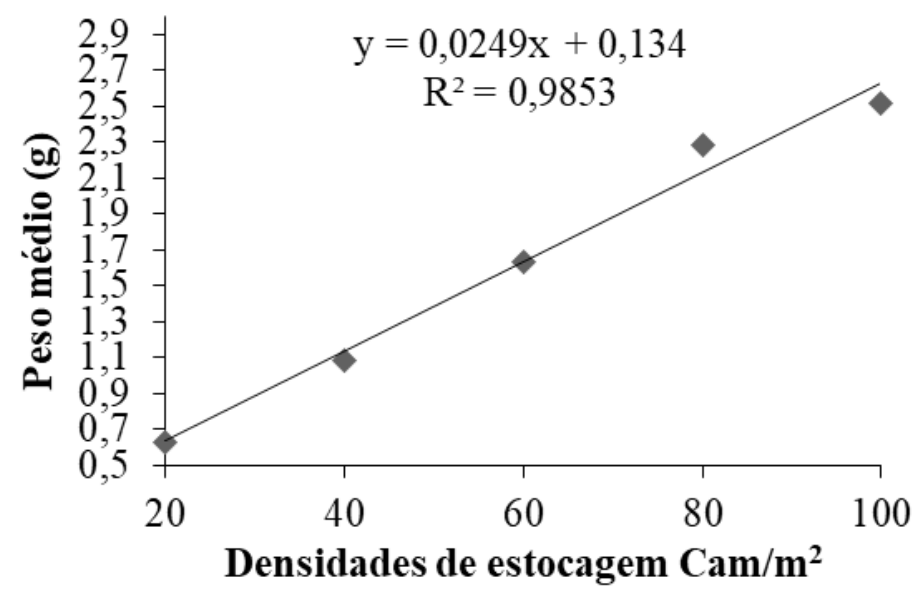

Figura 1. Relação entre o peso médio e a densidade do Macrobrachium amazonicum submetido a cinco tratamentos de diferentes densidades de estocagem.

Observou-se uma relação positiva com crescimento linear entre o peso médio do cultivo e a densidade (Figura 1). Levando em consideração que o presente trabalho teve duração de 30 dias, houve diferença significativa durante esse período para o M. amazonicum.

Em relação à sobrevivência (Figura 2), observou-se que quando há o aumento da densidade de estocagem de espécimes de Macrobrachium amazonicum, há um crescimento linear negativo. 


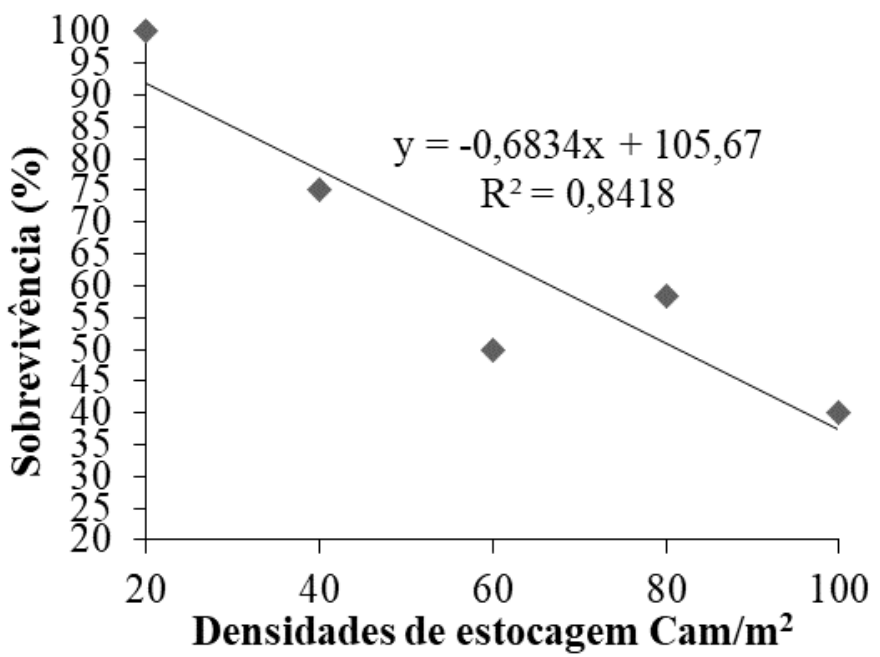

Figura 2. Relação entre a sobrevivência e a densidade de estocagem de espécimes de Macrobrachium amazonicum.

\section{DisCUSSÃo}

Segundo Maciel \& Valenti (2009), o M. amazonicum pode suportar uma variação alta de fatores abióticos. Porém, Sampaio, Silva, Santos \& Sales (2007) afirmam que essa espécie é encontrada em águas com oxigênio dissolvido de 4,6 a 6,1 mg L-1 e pH de 7,4 a 8,4, portanto apresenta valores um pouco abaixo dos encontrados no presente trabalho. No entanto, os valores médios das variáveis analisadas estiveram relativamente de acordo com o encontrado por Preto, Kimpara, Moraes-Valenti \& Valenti (2010) para cultivo de M. amazonicum.

Os resultados apresentados neste trabalho estão de acordo com os valores encontrados por Kiyohara (2006) em cultivo (7,5 meses) com M. amazonicum criados em tanques-rede, obtendo peso médio de $10 \mathrm{~g}$ na densidade de 20 camarões $/ \mathrm{m}^{2}$.

Segundo Rodrigues (2011), o cultivo de espécimes de M. amazonicum em viveiros protegidos por estufa no período de inverno em densidades de 10 e 20 camarões $/ \mathrm{m}^{2}$ pode ter influenciado no peso médio, pois após 60 dias de cultivo 2,76 $\pm 0,33 \mathrm{~g}$ e de 2,66 $\pm 0,26 \mathrm{~g}$, respectivamente, os valores corroboram o presente trabalho.

Maciel \& Valenti (2009), que utilizaram densidades de 40 e $80 \mathrm{cam} / \mathrm{m}^{2}$, observaram que os resultados dessas densidades de estocagens interferiram diretamente no desenvolvimento dos animais e, consequentemente, na sobrevivência, diminuindo a capacidade produtiva. Mas segundo Tidwell et al. (1997), o M. rosenbergii não apresentou diferença significativa na sobrevivência até o $2^{\circ}$ mês de cultivo em experimento semelhante ao ocorrido neste estudo.

Corroborando os resultados do presente trabalho, estão os experimentos de Silva (2007) e Moraes-Riodades \& Valenti (2004), que observaram que a densidade ideal para a produção de juvenis de $M$. amazonicum em cultivos superintensivos é de 80 camarões $/ \mathrm{m}^{2}$, proporcionando um 
desempenho produtivo aceitável, por haver um melhor aproveitamento da área e dos recursos.

É possível comparar as densidades trabalhadas por Negrini (2014) utilizando o camarão de água doce $M$. rosenbergii. De acordo com o autor, a produção de juvenis de $M$. rosenbergii é viável na densidade de 50 camarões $/ \mathrm{m}^{2}$, a qual apresentou a melhor sobrevivência, mostrando ter uma densidade próxima ao do cultivo de $M$. amazonicum, com valores de sobrevivência e taxa de conversão alimentar eficientes.

\section{CONCLUSÕES}

O cultivo de $M$. amazonicum é possível utilizando densidades entre 80 e 100 camarões $/ \mathrm{m}^{2}$ sem prejuízo do desempenho zootécnico, favorecendo a produção da espécie em cultivos com altas densidades. São necessários estudos com maior tempo de coleta de dados e que também determinem densidades específicas para cada modalidade de cultivo.

\section{REFERÊNCIAS}

ARAÚJO, M. C. \& VALENTI, W. C. (2005). Manejo alimentar de pós-larvas do camarão da Amazônia, Macrobrachium amazonicum, em berçário I. Acta Scientiarum, Animal Sciences, 27(1):67-72. http://dx.doi.org/10.4025/actascianimsci.v27i1.1243

FOOD AND AGRICULTURE ORGANIZATION OF THE UNITED NATIONS (FAO). (2016). FAO Statistical Yearbook: World Food and Agriculture. Rome: FAO. Acessado em abril. 2017. Disponível:< http://faostat.fao.org/

INSTITUTO BRASILEIRO DE GEOGRAFIA E ESTATÍSTICA (IBGE) (2015). Produção da Pecuária Municipal. Brasil: IBGE. v. 43.33 p.

KIYOHARA, F. (2006). Cultivo de Macrobrachium amazonicum (Heller, 1862) em cercados e em tanque-redes durante a fase de berçário e a fase de crescimento final e cultivo de Macrobrachium potiuna (Muller, 1880) em laboratório (Crustacea, Decapoda, Palaemonidae) [Tese de Doutorado]. São Paulo (SP): Instituto de Biociências, Departamento de Zoologia, Universidade de São Paulo.

KRUMMENAUER, D., WASIELESKY JR., W., CAVALLI, R. O., PEIXOTO, S. \& ZOGBI, P. R. (2006). Viabilidade do cultivo do camarão-rosa Farfantepenaeus paulensis (Crustácea, Decapoda) em gaiolas sob diferentes densidades durante o outono no sul do Brasil. Ciênc. Rural, 36(1):252257. http://dx.doi.org/10.1590/S0103-84782006000100039

MACIEL, C. R. \& VALENTI, W. C. (2009). Biology, Fisheries, and Aquaculture of the Amazon River Prawn Macrobrachium amazonicum: A Review. Nauplius, 17(2):61-79.

MARQUES, H. L. A. \& MORAES-VALENTI, P. M. C. (2012). Situação atual e perspectivas de cultivo do camarão gigante (Macrobrachium rosenbergii (De Man 1879) e do camarão do rio Amazonas Macrobrachium amazonicum (Heller 1862)) no Brasil. Aquacult. Res., 43:984-992. https://doi.org/10.1111/j.1365-2109.2011.03032.x 
MORAES-RIODADES, P. M. C. (2005). Cultivo do camarão-da-amazônia. Macrobrachium amazonicum (Heller, 1862) (Crustácea, Decapoda, Palaemonidae) em diferentes densidades: Fatores ambientais, biologia populacional e sustentabilidade econômica [Tese de Doutorado]. Jaboticabal (SP): Centro de Aquicultura, Universidade Estadual Paulista "Júlio de Mesquita Filho".

MORAES-RIODADES, P. M. C. \& VALENTI, W. C. (2004). Morphotypes in male Amazon river prawns, Macrobrachium amazonicum. 236:297-307. http://dx.doi.org/10.1016/j.aquaculture.2004.02.015

MORAES-VALENTI, P. \& VALENTI, V. C. (2010). Culture of the Amazon River Prawn Macrobrachium amazonicum. In: M. B. New, W. C. Valenti, J. H. Tidwell, L. R. D’abramo, M. N. Kutty (Eds.). Freshwater Prawns: Biology and Farming (pp.485-501). Oxford: Wiley-Blackwell.

NEGRINI, C. (2014) Criação de juvenis de camarão de água doce Macrobrachium rosenbergii em diferentes densidades de estocagem em sistema de bioflocos [Dissertação de Mestrado]. Palotina (PR): Departamento de Zootecnia, Universidade Federal do Paraná.

NEW, M. B. (2010). History and Global Status of Freshwater Prawn Farming. In: M. B. New, W. C. Valenti, J. H. Tidwell, L. R. D'abramo, M. N. Kutty (Eds.). Freshwater Prawns: Biology and Farming (pp.1-11). Oxford: Wiley-Blackwell.

PRETO, B. L., KIMPARA, J. M., MORAES-VALENTI, P. \& VALENTI, W. C. (2010). Population structure of pond-raised Macrobrachium amazonicum with different stocking and harvesting strategies. Aquaculture, 307:206-211. http://dx.doi.org/10.1016/j.aquaculture.2010.07.023

RODRIGUES, M. M. (2011). Efeito da alimentação e densidade de estocagem no desempenho zootécnico e no perfil celular do hepatopâncreas do camarão da-amazônia Macrobrachium amazonicum [Dissertação de Mestrado]. Jaboticabal (SP): Centro de Aquicultura, Universidade Estadual Paulista "Júlio de Mesquita Filho".

SAMPAIO, C. M. S., SILVA, R. R., SANTOS, J. A. \& SALES, S. P. (2007). Reproductive cycle of Macrobrachium amazonicum females (Crustacea, Palaemonidae). Braz. J. Biology, 67:551-559. http://dx.doi.org/10.1590/S1519-69842007000300022

SAS (1999). Software. Version 9.1. Cary, North Carolina: SAS Institute Inc.

SILVA, R. F. (2007). Efeito da densidade de estocagem durante a produção de juvenis do camarão de água doce Macrobrachium amazonicum em sistema de recirculação [Dissertação de Mestrado]. Curitiba: Universidade Federal do Paraná.

TIDWELl, J. H., COYLE, S. D., WEBSTER, C. D., SEDLACEK, J. D., WESTON P. A., KNIGHT, W. L., HILL JR., S. J., D’ABRAMO, L. R., DANIELS, W. H. \& FULLER, M. J. (1997). Relative prawn production and benthic macroinvertebrate densities in unfed, organically fertilized, and fed pond systems. Aquaculture, 149:227-242. https://doi.org/10.1016/S0044-8486(96)01452-4 